\title{
The Effect Of A Medical Decompression Protocol On The Use of Antiemetics, Nasogastric Tube Usage And Diet Advance In Late Stage Cancer Patients With Functional Intestinal Obstruction
}

\author{
Pedro H. Calves ${ }^{1 *}$, Cindy Kaye Saraceno ${ }^{1}$, Ann C. Eckardt Erlanger ${ }^{1}$,Patricia A. Eckardt ${ }^{2}$ \\ ${ }^{1}$ Good Samaritan Hospital Medical Center, West Islip, New York \\ ${ }^{2}$ Department of Nursing, Molloy College
}

Received: August 08, 2017; Accepted: November 09, 2017; Published: November 20, 2017

*Corresponding author: Pedro H. Calves, Good Samaritan Hospital Medical Center, Managing Director, West Islip, New York, Fax: 516-3233843; Tel: 516-323-3847 and E-mail: pedro.calves@chsli.org

\begin{abstract}
At the end of life, many patients suffer from a syndrome that simulates classic small bowel obstruction. The traditional approach to intestinal obstruction is relief of pressure in the gastrointestinal tract using mechanical methods such as naso-gastric tube insertion and suctioning. This relieves the distension of the hollow organs and thus the nausea.. Pharmacologic methods are also used in the care of these patients, however, there is debate as to which agents to use and when to use them.
\end{abstract}

The Medical Decompression protocol is a non-invasive pharmacologic approach to this problem. It brings with it ease and economy of delivery, the ability to administer it in intravenous as well as oral form, and is a multi-agent protocol of tried and true drugs used at the end of life: $1 / 2$ Metoclopramide, Dexamethasone, Diphenhydramine, and Pantoprazole

Prior to initiating this intervention, patients and their families are educated and are aware that this intervention is most likely a temporary measure aimed at symptom relief, as well as an attempt to improve quality of life.

Our sample contained 42 males and 62 females, with an average age of about 70 years. About $51 \%$ of participants received MD $(n=53)$. Results indicated that there was a statistically significant difference between the two groups related to days to progress diet.

We propose that a pharmacologic method such as MD is strongly considered as first line treatments for the functional intestinal obstruction syndrome and indeed be considered as adjuncts to allow the removal of invasive mechanical interventions in the end of life patient.

\section{Introduction}

Many patients with advanced cancer suffer from a condition that simulates classic small bowel obstruction. This often described but little studied para neoplastic syndrome goes by many names, (e.g.; Pseudo-Intestinal Obstruction, Ogilvie's
Syndrome) and manifests as abdominal pain, intractable nausea and unremitting vomiting. We use the term Functional Intestinal Obstruction (FIO) to describe this constellation of symptoms.

In a review of the literature, using data from the United States Department of Health and Human Services, it is estimated that in the United States approximately 62,000 patients are treated annually, in-hospital, for Functional Intestinal Obstruction with an average length of stay of 6 days [1]. Based on an average cost per hospital day of $\$ 1800.00$ this problem becomes a significant one in terms of the total burden on the health care system costing upwards of \$669 million a year [2].

For the individual patient and their family however, the premature loss of activity due to the debility of chronic nausea and vomiting prior to the patient's death is devastating, both in physical and emotional terms.

Traditional therapies include Nasogastric Tube (NGT) suctioning to relieve intraluminal pressure and volume upstream of the functional obstruction. Studies however, have shown that NGT placement with subsequent suctioning fails to relieve the symptoms of FIO in more than $86 \%$ of cases [3].

To alleviate the discomfort and potential side effects of NGT placements (i.e.: sinusitis, nasal irritation with epistaxis and nasal cartilage erosion, pain, increased incidence of aspiration pneumonia) Percutaneously Inserted Endoscopically Placed Gastrostomy (PEG) tubes have been used with varying degrees of success to alleviate the pressure and subsequent nausea associated with FIO.

Although successful in $83 \%-96 \%$ of patients in alleviating the nausea of FIO, PEG tubes cannot be placed in many cases [3]. This procedure also introduces additional cost, approximately $\$ 9600.00$ for insertion of PEG between practitioner and institutional charges and the social and psychological burden 
placed on the patient and family [4,5].Surgical Interventions are the sine qua non for relief of Intestinal Obstruction both Functional and Mechanical, however these procedures are indicated when patients have relatively good functional status, an expected lifespan of greater than 2 months and with a defined area of obstruction that can be relieved by diversion of the enteral stream [3]. Cost for surgical intervention ranges from $\$ 27,000$ to $\$ 57,000$ per procedure, not including the costs of any post-operative complications and ongoing supplies [3].Medical therapies have centered on the use of pro-motility, anti-secretory, and anti cholinergic agents to medically reduce the intraluminal pressure by reducing the production of intestinal secretions and to assist intestinal peristalsis [6].

While the syndrome of chronic pseudo-obstruction has been described in the literature, the full impact of the problem has not been outlined. For example, Laval, Arvieux, Stefani, Villard, Mestralet and Cadin placed FIO patients in a 3 tiered system with medications stratified [7]. The authors made a case for an organized strategy for this problem but did not introduce any new techniques.

Several authors have focused on the use of Octreotide, an inject able medication, along with corticosteroids and anti-emetics for relief of symptoms of FIO $[8,9]$. These reports regarding the effectiveness of various regimens including Octreotide had 15, 1, and 4 subjects respectively. Additionally, Octreotide is an inject able medication that is not outpatient friendly. The literature reveals no studies that investigate the effectiveness of our MD Regimen.

The purpose of this study was to examine the effect of the MD protocol.MD consists of $1 / 2$ Metoclopramide (Reglan ${ }^{\mathrm{TM}}$, Teva, USA Pharmaceuticals) 10mg IVP every six hours, Dexamethasone (Decadron $^{\mathrm{TM}}$, Westword, USA) 4mg IVP every six hours, Diphenhydramine (Bendryl ${ }^{\mathrm{TM}}$, APP Pharmaceuticals) 25mg every eight hours, and Pantoprazole (Protonix ${ }^{\mathrm{TM}}$, Novaplus, USA) $40 \mathrm{mg}$ IV daily.

\section{Method}

\section{Participants}

There were 42 males (about 40\%of the sample) and 62 females (roughly $60 \%$ of the sample), with a mean age of 70 years $(S D=13.69)$, ranging from 35 years to 97 years of age. The sample size in each group were equivalent, as $51 \%$ of participants received MD $(n=53)$, and $49 \%$ of participants did not receive MD $(n=51)$, for a total sample size of 104 participants. The majority of participants (about 70\%, $n=73$ ) had an NG tube previously, while just under $30 \%$ of the sample $(n=31)$ did not.

Of those participants following a medically directed prior to admission (22\%of the sample, $n=23$ ) most had a clear liquid diet. Please see table 1 [Table 1].

Prior to admission, the majority of participants had NG tubes, reported nausea and vomiting, and were antiemetic. Please see table 2 [Table 2].

There were 10 primary cancer diagnoses for all patients $(\mathrm{n}=$ 104). Please see table 3 [Table 3].

\begin{tabular}{|c|c|c|}
\hline Table 1: Pre-admission diet & \multicolumn{2}{|l|}{} \\
\hline Diet & $\mathbf{n}$ & $\mathbf{\%}$ \\
\hline Clear liquid & 13 & 12.5 \\
\hline Soft & 6 & 5.8 \\
\hline Regular & 4 & 3.8 \\
\hline $\mathrm{n}=23$ & & \\
\hline
\end{tabular}

\begin{tabular}{|c|c|c|}
\hline \multicolumn{2}{|c|}{ Table 2: preadmission status } \\
\hline Status & No & Yes \\
\hline NG & $29.8 \%(31)$ & $70.28 \%(73)$ \\
\hline Nausea & $3.8 \%(4)$ & $96.28 \%(100)$ \\
\hline Vomit & $11.5 \%(12)$ & $88.58 \%(92)$ \\
\hline Antiemetic & $15.4 \%(16)$ & $84.68 \%(88)$ \\
\hline
\end{tabular}

Notes: Number in parentheses indicates $\mathrm{n}$.

$\mathrm{NG}=$ Nasogastric Tube

\begin{tabular}{|c|c|c|}
\hline Table 3: Cancer diagnoses & \\
\hline Type & $\mathbf{n}$ & \% \\
\hline bladder & 2 & 1.9 \\
\hline breast & 2 & 1.9 \\
\hline colon & 46 & 44.2 \\
\hline kidney & 1 & 1 \\
\hline liver & 7 & 6.7 \\
\hline lung & 9 & 8.7 \\
\hline ovary & 23 & 22.1 \\
\hline pancreas & 6 & 5.8 \\
\hline stomach & 1 & 1 \\
\hline uterus & 7 & 6.7 \\
\hline
\end{tabular}

The most common comorbidity was multiple conditions ( $\mathrm{n}=$ 38 , about $37 \%$ of the sample). For singular or no comorbidities, the most common were as follows: cardiac $(n=32,31 \%$ of the sample), no comorbidity ( $\mathrm{n}=25,24 \%$ of the sample), and then pulmonary, neuromuscular, or diabetes (all $n=3$, about $3 \%$ of the sample).

\section{Procedure}

Medical records were deemed eligible if patients were: aged 18 years, had a diagnosis of functional bowel obstruction, had documented abdominal pain, intractable nausea \&/or vomiting, and advanced stage GI or reproductive tract cancer. Records were excluded if for complete intestinal obstruction on radiological examination. Eligible cases prior to April 2007 (no MD, which was first implemented in April 2007) and charts dated after April 2007 (received the $M D$ regimen) were compared using t-tests on the outcome variable days to advance diet. 


\section{Results}

A mean of 0.08 doses $(S D=.56)$ were required to resolve symptoms. It took a mean of 4.6 days to advance diet $(S D=3.84)$.

A t-test was conducted to investigate significant differences in means between the two group. Results indicated that there was a statistically significant difference between the medical decompression group $(M=1.30, S D=.93)$ and the non-medical decompression group $(M=4.60, S D=3.84)$ related to days to progress diet $\mathrm{t}(54.43)=5.91, \mathrm{p}=<.01$.

To ensure the two groups were equivalent, and to allow for more robust causal inference, propensity score matching estimates were utilized. Please see figure 1[Figure 1].

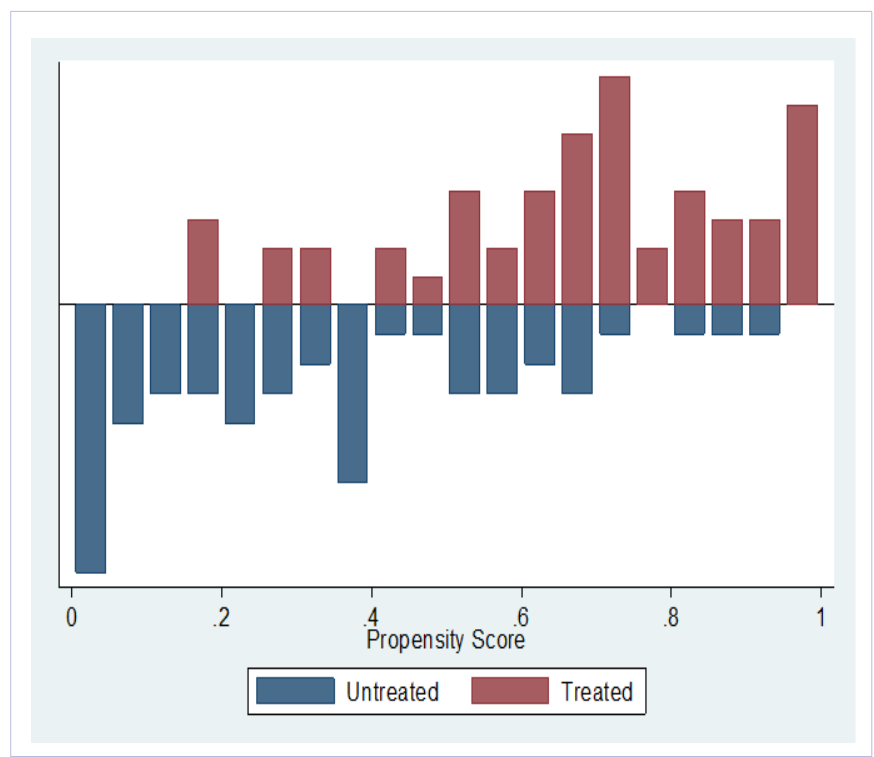

Figure 1:Propensity Score Matching

Participants that were not matched $(n=23)$ were removed. These participants were all males with an average age of about 76 years $(S D=12)$, and none had an NG tube inserted previously. While over half did not have data on diet prior to admission, (n $=13,56.5 \%$ of the sample), $43.5 \%$ of participants $n=10$, were on a clear liquid diet pre-admission. None reported nausea or vomiting before admission.

A follow-up t-test ( $n=81$ ) was conducted. Results indicated that there was a statistically significant difference between the medical decompression group $(M=1.39, S D=.92)$ and the nonmedical decompression group $(M=3.46, S D=2.60)$ related to days to progress diet $t(43.59)=4.62, p=<.01$

\section{Discussion}

The statistical analysis bears out what the team observed at bedside, that there is a significant improvement in the ability to advance the diet in these patients. This represents a decreased reporting of nausea and subsequent decreased use of anti emetics. In $M D$, patients the time from initiation of the protocol to the advancement in diet was 4.6 days. In the equivalent control group this number was 5.91 days.
This decrease of 1.3 days in time to the improvement of patient symptoms leading to advancement of diet represents an increase in patient and family satisfaction, decrease in acute care length of stay and, additional cost savings to the system due to a decreased need for surgical intervention in this patient population.

No studies have been found in the literature that addresses this symptom complex with this simple and relatively inexpensive regime. We believe that additional studies should be conducted to investigate not merely the effectiveness of the $M D$ protocol, but the potential cost savings to the Health Care System and effect on patient and family satisfaction at the end of life.

If these studies replicate our findings, and the suffering caused by these frequent sequelae of terminal illness can be relieved, $M D$ could be added to the list of "Gold" standards for Palliative Care therapies.

\section{References}

1. Centers for Disease Control and Prevention. US Dept. of Health and Human Services. 2010.

2. Henry J. The Kaiser Family Foundation Stat Health Facts. Data Source: Hospital In-Patient Day Expenses.

3. Roeland E, von Gunten CF. Current concepts in malignant bowel obstruction management. Curr Oncol Rep. 2009; 11(4): 298-303.

4. Callahan CM, Buchanan NN, Stump TE. Healthcare Costs Associated With Percutaneous Endoscopic Gastrostomy Among Older Adults In A Defined Community. J Am Geriatr Soc. 2001; 49(11):1525-1529.

5. Ekberg O, Hamdy S, Woisard V, Wuttge-Hannig A, Ortega P. Social And Psychological Burden Of Dysphagia: It's Impact On Diagnosis and Treatment. Dysphagia. 2002; 17(2):139-146; Doi: 10.1007/ s00455-001-0113-5

6. Weber C,Zulian GB.Malignant Irreversible Intestinal Obstruction:The Powerful Association of Octreotide to Corticosteroids, Antiemetics, and Analgesics. The American Journal of Hospice and Palliative Care. 2009; 26(2): 84-88; Doi: 10.1177/1049909108327967

7. Laval G, Arvieux C, Stefani L, Villard ML, Mestrallet JP, Cardin N. Protocol for the Treatment of Malignant Inoperable Bowel Obstruction: A Prospective Study of 80 Cases at Grenoble University Hospital Center. J Pain Symptom Manage. 2006; 31(6) :502-512; Doi: 10.1016/j.jpainsymman.2005.10.009

8. Mercadante S, Ferrera P, Villari P, Marrazzo A. Aggressive pharmacological treatment for reversing malignant bowel obstruction. J Pain Symptom Manage. 2004; 28(4): 412-416; Doi: 10.1016/j.jpainsymman.2004.01.007

9. Myers J, Tamber A, Farhadian M. Management of Treatment-Related Intermittent Partial Small Bowel Obstruction: The Use of Octreotide. J Pain Symptom Manage. 2010; 39(4): e1-3; Doi: 10.1016/j. jpainsymman.2009.11.309

10. Microsoft Excel. Redmond, Washington. Computer Software. Microsoft. 2007.

11. IBM Corp. IBM SPSS Statistics for Windows, Version 24.0. Armonk, NY: IBM Corp. Released 2016 